\title{
Ausbildungsziele für Sozialpädiatrie
}

M. Hämmerli und J.-C. Vuille

Institut für Sozial- und Präventivmedizin der Universität Bern, Inselspital, 3010 Bern, und Schularztamt der Stadt Bern, Monbijoustrasse 11, 3011 Bern.

VORGEHEN :

LEBENSBEREICHE

DES KINDES

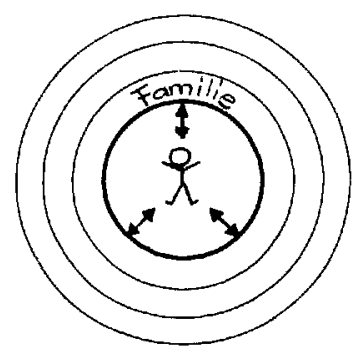

Säugling, Kleinkind

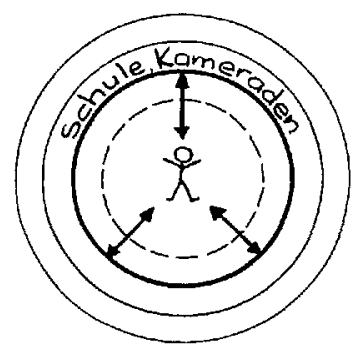

Schulkind

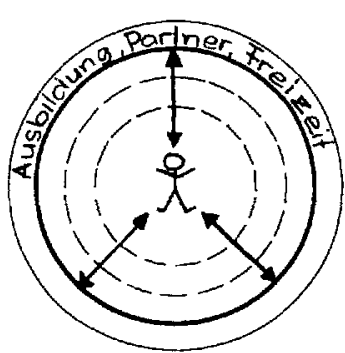

Jugendlicher

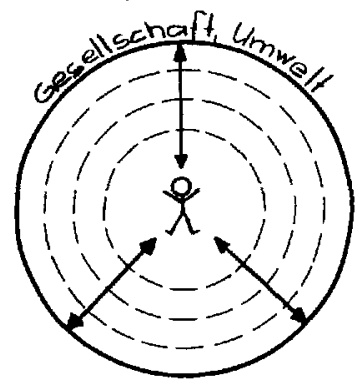

alle Altersstufen

- ERARBEITEN EINER PROBLEMLISTE ANHAND DER LITERATUR

- PROVISORISCHER LERNZIELKATALOG

- INTERDISZIPLINÄRE VERNEHMLASSUNG

- DEFINITIVER LERNZIELKATALOG

- REALISIERUNG IM UNTERRICHT DER PÄDIATRIE UND DER SOZIAL- UND PRÄVENTIVMEDIZIN

\section{MÖGLICHE \\ STÖRFAKTOREN}

ERNÄHRUNGS- UND

PFLEGEPROBLEME

ENTWI CKLUNGSSTÖRUNGEN

ERZI EHUNGSSCHWIERIGKEI TEN

SCHULPROBLEME

VERHALTENSSTÖRUNGEN

BEHINDERUNGEN,

CHRONISCHE KRANKHEITEN

SUIZIDGEFÄHRDUNG

SUCHTMI TTELKONSUM

PROBLEME DER SEXUALITÄT

GESUNDHEITSSCHÄDI GENDES

VERHALTEN

UNFALLGEFÄHRDUNG

SOZI OÖKONOMISCHE RISI KOFAKTOREN
AUFGABENBEREICHE DER SOZIALPÄDIATRIE

MÜTTERBERATUNG

VORSORGEUNTERSUCHUNGEN

ELTERNB I LDUNG

SCHULÄRZTLICHER DIENST

ERZI IEHUNGSBERATUNG

SONDERSCHULEN

KR I SENINTERVENTION

BERATUNGSSTELLEN

FAMILIENPLANUNG

GESUNDHEITSERZ IEHUNG

UMWELTGESTALTUNG

EPIDEMI OLOG ISCHE

FORSCHUNG 